\title{
Fuzzy Ontologies for Specialized Knowledge Representation in WordNet
}

\author{
Fernando Bobillo ${ }^{1}$, Juan Gómez-Romero ${ }^{2}$, and Pilar León Araúz ${ }^{3}$ \\ 1 Department of Computer Science and Systems Engineering, \\ University of Zaragoza, Spain \\ fbobillo@unizar.es \\ 2 Department of Computer Science, Applied Artificial Intelligence Group, \\ University Carlos III of Madrid, Spain \\ jgromero@inf .uc3m.es \\ 3 Department of Translation and Interpreting, University of Granada, Spain \\ pleon@ugr.es
}

\begin{abstract}
Despite the undisputed success of ontologies, they are not appropriate to represent and reason with vague knowledge. WordNet, a widely used ontology for general/natural language tasks, presents some of these problems when it is applied to specialized discourse. In this paper, we propose to use fuzzy ontologies, which combine Fuzzy Logic theory and Description Logics, to represent the imprecise notions of prototypicality and representativeness inside a synonym set, semantic similarity, and hyponymic degree in WordNet. We show that this approach is particularly appropiate to combine WordNet with other specific terminological resources, such as the environmental knowledge base EcoLexicon.
\end{abstract}

\section{Introduction}

In the last decade, ontologies have become the state-of-the-art knowledge representation formalism. An ontology is an explicit and formal specification of the concepts, individuals and relationships that exist in some area of interest, created by defining axioms that describe the properties of these entities [1]. The adoption of ontologies by the Semantic Web community has resulted in the development of ontology languages, such as RDF (Resource Description Framework) and OWL 2 (Ontology Web Language, version 2), which are the current standards for metadata representation in the next-generation Web.

WordNet is a lexical database for the English language created at the University of Princeton 7. It groups nouns, verbs, adjectives and adverbs into sets of cognitive synonyms (named synsets) which each one expresses a different concept. Synsets are related through semantic relations, composing a semantic network of concepts designated by various synonym words (actually, word senses). WordNet is publicly available, and can be accessed with a web browser or downloaded as a standalone database application 1 . It has been also translated into RDF and OWL to be used in the Semantic Web [12].

\footnotetext{
${ }^{1}$ http://wordnet.princeton.edu
} 
WordNet has been successfully used as a semantic thesaurus in computational linguistics and natural language processing. It can be applied to improve information retrieval tasks from text sources: term extraction, word disambiguation, query expansion with synonym terms, automatic annotation of texts, etc. Nevertheless, WordNet is not intended for specialized text processing, since it only includes general language knowledge [3. This problem mostly affects the synsets defined in the leaf nodes of the WordNet taxonomy, since they group together word senses that might be considered synonyms by lay users, but have different meanings and cannot be interchanged in a specialized context. In these cases, specialized resources are required, such as EcoLexicon 22. EcoLexicon is a terminological knowledge base that represents the conceptual structure of the specialized domain of the Environment [5]. Unfortunately, specialized resources are not usually connected to WordNet, thus missing its support for the analysis of the common discourse.

The exploitation of WordNet in specialized text processing requires extending the representation to set imprecise connections between some pieces of information, rather than using WordNet's sharp equivalences and relations between terms. However, despite the undisputed success of ontologies, they are not appropriate to deal with imprecise and vague knowledge. In that regard, proposals for the creation of fuzzy ontologies have emerged [6]. In fuzzy ontologies, concepts denote fuzzy sets and relations denote fuzzy relations; therefore, the axioms are not in general either true or false, but they may hold to some degree of truth.

In this paper, we study the limitations of WordNet for specialized knowledge representation, and propose an extension based on fuzzy ontologies to overcome them. Previous research has focused on the use of WordNet for the automatic creation of fuzzy ontologies 4. However we focus on the inherent imprecision of WordNet contents and, in particular, on their representation with fuzzy ontologies, which has not been explored to date. We show that the fuzzification of the hyponymOf, hypernymOf, inSynset and containsWordSense relations is helpful to: (1) discriminate between the several word senses included in the same WordNet synset; and (2) provide a more fine-grained representation of the semantic distance between a concept and its hyponyms/hypernyms. These two features would allow the integration of EcoLexicon specialized information into a WordNet schema without loss of knowledge.

The remainder of this paper is structured as follows. In the next section, we briefly present the structure and some limitations of WordNet. In Section 3. we show how the fuzzy WordNet representation is created and which reasoning tasks can be performed. These contributions are illustrated by showing an example based on EcoLexicon knowledge. The paper concludes with a discussion on the results and some comments about prospective directions for future work.

\section{Limitations of WordNet in Specialized Knowledge Representation}

The basic elements in the WordNet knowledge base are words, word senses and synsets. Words correspond to terms; the lexical form is the written expression

\footnotetext{
2 http://ecolexicon.ugr.es
} 
of the word. Since the same word can be used with different senses in different pragmatic contexts, a word has several associated senses - a sense is associated to a single word. According to WordNet specification, a synset is a collection of word senses that can be considered as synonyms -hence, a word sense is included into one synset at most. Each synset is associated with a gloss, which is a brief textual description of the meaning of the synset. Synsets are interlinked by means of semantic relations. In this paper, we will focus on the hyponymic relations hyponymOf and its inverse hypernymOf, which represent the notions of subtype-of and supertype-of, respectively, and on the relations inSynset and its inverse containsWordSense, which link words and synsets. Figure 1 represents these core elements of WordNet.

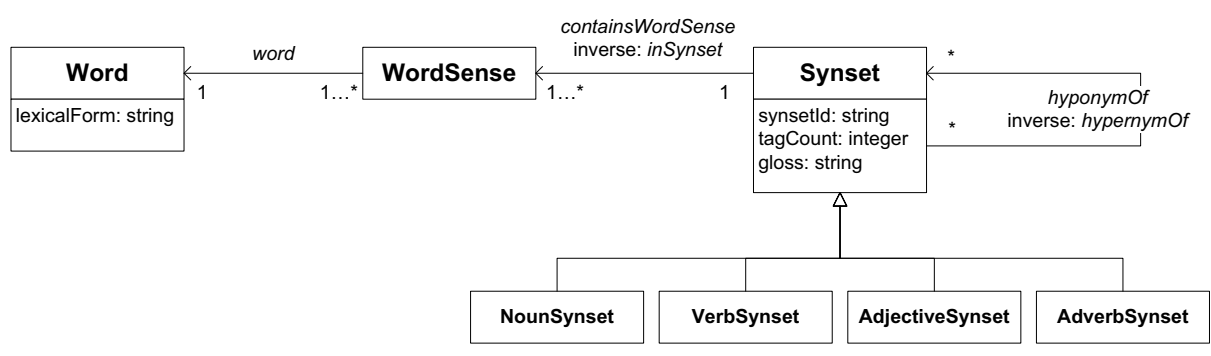

Fig. 1. UML schema of the core WordNet entities

Figure2 2 shows the definition of the synset $S 1$ \{ $\underline{\mathrm{knoll}}, \underline{\text { mound }}$, hillock, hummock, $\underline{\text { hammock }}\}$, its hypernym $S 0\{\underline{\text { hill }}\}$, and its hyponyms $S 2, S 3$ and $S 4$. The gloss of each synset is also included. Note that the elements in the synset are not words, but specific word senses associated with the words. We will use the lexical form of the associated word to notate a word sense, as it is done in WordNet.

So: (n) hill (a local and well-defined elevation of the land) "they loved to roam the hills of West Virginia"

S1: (n) knoll, mound, hillock, hummock, hammock (a small natural hill)

$S 2$ : (n) anthill , formicary (a mound of earth made by ants as they dig their nest)

$S 3$ : (n) kopje, koppie (a small hill rising up from the African veld)

S4: (n) molehill (a mound of earth made by moles while burrowing)

Fig. 2. WordNet representation of synsets (synset identificators are ours)

The RDF/OWL translation proposed by the WordNet Task Force of the World Wide Web Consortium can be considered the official ontology-based version of WordNet [12]. The WordNet ontology defines three main classes: Word, WordSense and Synset. Concrete elements included in WordNet are included as instances of these classes. Relations have also been instantiated to mirror WordNet associations. Note that this translation does not treat WordNet as a class hierarchy where the hyponym relationship is interpreted as a class inclusion axiom. 
While this is possible by adding a few axioms to the ontology, the semantics of the hyponym relation in WordNet discourages this interpretation.

The use of WordNet in specialized contexts, either isolated or in combination with domain-specific terminological knowledge bases, is not a straight-forward task. As several researchers have claimed [8], synsets and their relations have not always been created according to the same criteria, and for specialized terms, they may not be consistent. Particularly, for the case of EcoLexicon, the representation of specialized knowledge needs a more fine-grained approach in terms of categorization. In the following examples, we will show how both synset members and hyponym synsets should not have the same status of representativeness.

Theoretically, the notion of synset corresponds to that of meaning, and ultimately to that of a distinct concept, since all word senses included in a synset are close enough to represent the same concept in reality. Nevertheless, synset members show different degrees of similarity.

In Figure 2, synsets $S 2$ \{anthill, formicary\} and $S 3\{$ kopje, kopiie $\}$ are composed of two word senses designated by clear synonyms. They undoubtedly represent the same concept in reality. Thus, they are equivalent senses which share the highest degree of similarity.

In synset $S 1\{\underline{\mathrm{knoll}}, \underline{\text { mound}}, \underline{\text { hillock}}, \underline{\text { hummock}}$, hammock $\}$, word senses are still close concepts that share meaning identity, but their similarity degree differs slightly from that of synsets $S 2$ and $S 3$. We could then say that some of these word senses (i.e. knoll) are closer (or more similar) than others (i.e. hammock) to the prototype of small hill, the meaning represented in the synset.

In the same way, it is also evident that either synset $S 2$ or synset $S 4$ \{molehill are more accurately types of mounds than knolls, whereas synset $S 3$ is better categorized as a knoll than as a hammock. Consequently, there is also a clear relationship between the individual word senses of a synset and the word senses of its hyperonym synset. Nevertheless, WordNet does not represent it, since this is not necessarily important for general language purposes. Equally, from the three synsets that constitute the hyponyms of synset $S 1$, anyone would easily point synset $S 3$ as the most prototypical exemplar of a small natural hill.

Prototype theory [10] provides an approach to account for such effects of prototypicality on categorization. Prototype theory is based on the fact that concepts are graded. They show different degrees of category membership according to the notions of family resemblance [13] and similarity. From the 70s there has been a lively discussion regarding the suitability of fuzzy sets within the scope of the prototype theory. Initially they were widely accepted and applied by the psychology of concepts, but in the 80 s cognitivists started to reject them in the realm of conceptual compositionality [9]. However, Zadeh showed that it was very unlikely that an adequate theory of prototypes could be constructed without an explicit use of fuzzy sets and related concepts [14].

Synsets should not be regarded as a mere set of more or less canonical synonyms. They are not even the even union of all word senses. Rather, they are prototypical categories with fuzzy boundaries whose members (senses) belong to it in different degrees. In our approach, both intra-synsets and hyponymic 
S5: (n) breakwater, groin, grovne, $\underline{\text { mole }}, \underline{\text { bulwark, }}$ seawall, jetty (a protective structure of stone or concrete; extends from shore into the water to prevent a beach from washing away)
S6: (n) offshore breakwater, detached breakwater
$S 7:$ (n) convex seawall, rigid revetment
S8: (n) fish-tail groin, $\underline{\text { Y-shaped groin }}$

Fig. 3. WordNet $(S 5)$ and EcoLexicon $(S 6, S 7, S 8)$ combined synsets

relations (either among word senses or synsets) are regarded as fuzzy constructs that rely on different levels of prototypicality according to the concept of similarity. After all, similarity is the common cognitive feature involved in category membership, synonymy and hyponymy construction. This is especially important for the integration of specialized knowledge in WordNet, since some of the concepts contained in EcoLexicon as distinct notions are compiled in WordNet within the same synset.

In synset $S 1$ although word senses were not equally prototypical, they shared the same meaning identity. In synset $S 5$ (Figure 3), however, they do not point at all to the same concept in reality. In this case, the only true synonyms in the synset are groin and groyne. It is our guess that WordNet displays all of the concepts within the same synset due to its general language approach. Nonetheless, if we were to integrate all kinds of coastal protective structures from EcoLexicon degrees would be vital for the development of its hyponym synsets.

In $S 5$ the prototype actually corresponds to one of its members. Groin (and groyne) can be regarded as the prototype because its definitional features match the gloss of the synset. Therefore, groyne and groin are related to their own synset with the maximum degree. Consequently, bulwark, which is the concept less related to the gloss (and thus, less similar to groin), has the lower degree in terms of synset membership.

Following the general-language perspective of WordNets synsets, we have included what could be three different hyponymic synsets extracted from EcoLexicon (Figure 31). Synset $S 8$ is composed of two word senses that are fully synonymic and thus should have the same synset membership degree. Synsets $S 6$ and $S 7$ are not strict synomyms but, according to metalinguistic information in lexicographic resources, there is often no distinction between offshore and detached breakwaters and convex seawalls and rigid revetments. This is why they belong to the same synset but should not have the same degree of prototypicality. In Section 3 we show how to construct such representation with fuzzy ontologies and provide an example on degree assignment and reasoning with synset membership and hyponymic relations.

\section{$3 \quad$ Fuzzy Extension of WordNet}

\subsection{Fuzzy Ontologies}

The main formalism behind ontology languages are Description Logics (DLs), a family of logics for representing structured knowledge. Each logic is denoted by 
Table 1. Syntax and semantics of the fuzzy DL $\mathcal{S} \mathcal{R}^{-} \mathcal{I}$

\begin{tabular}{|c|c|c|}
\hline \multicolumn{2}{|c|}{ Concept Syntax $(C)$} & \multirow{2}{*}{$\begin{array}{l}\text { Semantics }\left(C^{\mathcal{I}}(x)\right) \\
A^{\mathcal{I}}(x)\end{array}$} \\
\hline$\overline{(\mathrm{C} 1)}$ & $A$ & \\
\hline$(\mathrm{C} 2)$ & $\top$ & $\gamma_{0}$ \\
\hline (C3) & $\perp$ & $\gamma_{p}$ \\
\hline (C4) & $\bar{C} \sqcap D$ & $C^{\mathcal{I}}(x) \otimes D^{\mathcal{I}}(x)$ \\
\hline (C5) & $C \sqcup D$ & $C^{\mathcal{I}}(x) \oplus D^{\mathcal{I}}(x)$ \\
\hline (C6) & $\neg C$ & $\ominus C^{\mathcal{I}}(x)$ \\
\hline (C7) & $\forall R . C$ & $\inf _{y \in \Delta^{\mathcal{I}}}\left\{R^{\mathcal{I}}(x, y) \Rightarrow C^{\mathcal{I}}(y)\right\}$ \\
\hline (C8) & $\exists R . C$ & $\sup _{y \in \Delta^{\mathcal{I}}}\left\{R^{\mathcal{I}}(x, y) \otimes C^{\mathcal{I}}(y)\right\}$ \\
\hline Role & Syntax $(R)$ & Semantics of $R^{\perp}(x, y)$ \\
\hline$\overline{(\mathrm{R} 1)}$ & $R$ & $R^{\mathcal{I}}(x, y)$ \\
\hline$(\mathrm{R} 2)$ & $R^{-}$ & $R^{\mathcal{I}}(y, x)$ \\
\hline Axiom & Syntax $(\tau)$ & Semantics $(\mathcal{I}$ satisfies $\tau$ if ...) \\
\hline$\overline{(\mathrm{A} 1)}$ & $\langle a: C \bowtie \alpha\rangle$ & $C^{\mathcal{I}}\left(a^{\mathcal{I}}\right) \bowtie \alpha$ \\
\hline$(\mathrm{A} 2)$ & $\langle(a, b): R \geq \alpha\rangle$ & $R^{\mathcal{I}}\left(a^{\mathcal{I}}, b^{\mathcal{I}}\right) \geq \alpha$ \\
\hline (A3) & $\left\langle C_{1} \sqsubseteq C_{2} \geq \alpha\right\rangle$ & $\inf _{x \in \Delta^{\mathcal{I}}}\left\{C_{1}^{\mathcal{I}}(x) \Rightarrow C_{2}^{\mathcal{I}}(x)\right\} \geq \alpha$ \\
\hline (A4) & $\left\langle R_{1} \ldots R_{m} \sqsubseteq R \geq \alpha\right\rangle$ & $\begin{array}{l}\inf _{x_{1}, x_{n+1} \in \Delta^{\mathcal{I}}}\left\{\sup _{x_{2} \ldots x_{n} \in \Delta^{\mathcal{I}}\{}\right. \\
\left.\left.\left.R_{1}^{\mathcal{I}}\left(x_{1}, x_{2}\right) \otimes \cdots \otimes R_{n}^{\mathcal{I}}\left(x_{n}, x_{n+1}\right)\right\}\right\} \Rightarrow R^{\mathcal{I}}\left(x_{1}, x_{n+1}\right)\right) \geq \alpha\end{array}$ \\
\hline
\end{tabular}

using a string of capital letters which identify the expressivity of the logic. For instance, the standard language for ontology representation OWL 2 is equivalent to $\mathcal{S} \mathcal{R O} \mathcal{I} \mathcal{Q}(\mathbf{D})$. Fuzzy DLs are the extension of DLs in the fuzzy case. Essentially, in fuzzy ontologies fuzzy concepts denote fuzzy sets of individuals, whereas fuzzy roles (or properties) denote fuzzy binary relations among individuals. For the sake of clarity, in the rest of this paper we will consider a relatively simple fuzzy DL, fuzzy $\mathcal{S} \mathcal{R}^{-} \mathcal{I}$, which is a fragment of fuzzy $\mathcal{S} \mathcal{R O} \mathcal{I} \mathcal{Q}(\mathrm{D})$ [1].

A fuzzy Knowledge Base (KB) or fuzzy ontology contains a finite number of axioms stating information about fuzzy concepts (or classes) (denoted $C, D$ ), fuzzy roles (or relations, denoted $R$ ) and individuals (denoted $a, b$ ). Fuzzy concepts can be atomic or complex, inductively formed from other concepts. Roles can be atomic or the inverse of a role. Table 1 shows the concepts, roles and axioms of fuzzy $\mathcal{S} \mathcal{R}^{-} \mathcal{I}$, where $\bowtie \in\{\geq, \leq\}, \alpha$ is a degree of truth, $\otimes$ is a t-norm, $\ominus$ is a negation, $\oplus$ is a t-conorm, and $\Rightarrow$ is an implication. Usually, the set of degrees of truth $\mathcal{N}$ is assumed to be $[0,1]$. Instead, we will assume that the set of degrees of truth $\mathcal{N}$ is a finite ordered set $\left\{\gamma_{0}, \gamma_{1}, \ldots, \gamma_{p}\right\}$ with the minimum value $\gamma_{0}$ representing absolute falsity, and the maximum value $\gamma_{p}$ representing absolute truth. We assume that the operators $\otimes, \oplus, \ominus, \Rightarrow$ are those of Zadeh fuzzy logic except in axioms (A3) and (A4) which use Gödel implication [1].

The semantics of the logic is given by a fuzzy interpretation. A fuzzy interpretation $\mathcal{I}$ is a pair $\left(\Delta^{\mathcal{I}},{ }^{\mathcal{I}}\right)$ consisting of a non empty set $\Delta^{\mathcal{I}}$ (the interpretation domain) and a fuzzy interpretation function ${ }^{\mathcal{I}}$ mapping: (i) an individual $a$ to an element $a^{\mathcal{I}} \subseteq \Delta^{\mathcal{I}}$, (ii), a fuzzy concept $C$ to a function $C^{\mathcal{I}}: \Delta^{\mathcal{I}} \rightarrow \mathcal{N}$, and a fuzzy role $R$ to a function $R^{\mathcal{I}}: \Delta^{\mathcal{I}} \times \Delta^{\mathcal{I}} \rightarrow \mathcal{N} . C^{\mathcal{I}}$ (resp. $R^{\mathcal{I}}$ ) denotes the membership function of the fuzzy concept $C$ (resp. fuzzy role $R$ ) w.r.t. $\mathcal{I} . C^{\mathcal{I}}\left(a^{\mathcal{I}}\right)$ 
(resp. $R^{\mathcal{I}}\left(a^{\mathcal{I}}, b^{\mathcal{I}}\right)$ ) gives us to what extent the individual $a$ can be considered an element of the fuzzy concept $C$ (resp. to what extent $(a, b)$ can be considered an element of the fuzzy role $R$ ) under the fuzzy interpretation $\mathcal{I}$.

There are several reasoning algorithms for fuzzy ontologies. We will focus on a family of algorithms called crisp representation algorithms. Given a fuzzy ontology, crisp representation algorithms solve the reasoning tasks by computing an equivalent OWL 2 ontology. This makes it possible to reuse existing reasoners, editors, etc. The interested reader can find the details of the process in 1 . DELorEAnis an implementation of a crisp representation algorithm for Fuzzy OWL 2. For implementation details, we refer the reader to [2].

\subsection{Fuzzy WordNet Ontology}

According to our discussion in Section 2, we propose a new representation for the integration of WordNet and EcoLexicon knowledge:

- We assume that both containsWordSense and its inverse relation inSynset are fuzzy, as the membership of a sense to a synset is a matter of degree.

- Similarly, hyponymOf and its inverse relation hypernymOf are fuzzy now.

- We define a new fuzzy transitive relation subsenseOf between senses, representing the degree to which a sense is a subtype of another one.

- We add the following axiom:

$$
\text { 〈containsWordSense subsenseOf inSynset } \sqsubseteq \text { hyponymOf } \geq 1 \text { > }
$$

Equation 1 makes it possible to compute a lower bound for the degree of a synset sy1 being an hyponym of a synset sy2. In fact, the semantics implies that:

$$
\begin{aligned}
(\text { hyponymOf })^{\mathcal{I}}(\text { sy } 1, \text { sy } 2) & \geq \sup _{\text {sense } 1, \text { sense } 2 \in \Delta^{\mathcal{I}}}\{\text { containsWordSense } \\
& \text { subsenseOf } f^{\mathcal{I}}(\text { sense } 1, \text { sense } 1) \otimes \\
& \text { sense } \left.) \otimes \text { inSynset }^{\mathcal{I}}(\text { sense2 } 2, \text { sy } 2)\right\}
\end{aligned}
$$

Note that if there exist several senses related through the property subsenseOf, the maximum degree of truth is considered.

Example 1. Consider the following fragment of a fuzzy $\mathrm{KB} \mathcal{K}$, assuming the values for $\alpha_{i, j}$ and $\beta_{k, S_{l}}$ assigned by domain experts and depicted in Figure 4.

$$
\begin{array}{r}
\left\{\left\langle\left(\text { sense }_{\mathrm{i}}, \text { sense }_{\mathrm{j}}\right): \text { subsenseOf } \geq \alpha_{i, j}\right\rangle: \forall i \in\{8, \ldots, 13\}, \forall j \in\{1, \ldots, 7\}\right\} \bigcup \\
\left\{\left\langle\left(\text { sense }_{\mathrm{k}}, \mathrm{S}_{\mathrm{l}}\right): \text { inSynset } \geq \beta_{k, S_{l}}\right\rangle: \forall k \in\{1, \ldots, 13\}, \forall l \in\{5, \ldots, 8\}\right\}
\end{array}
$$

It holds that (hyponymOf) $)^{\mathcal{I}}(\mathrm{S} 6, \mathrm{~S} 5) \geq 0.9$, (hyponymOf) $)^{\mathcal{I}}(\mathrm{S} 7, \mathrm{~S} 5) \geq 0.6$ and (hyponymOf $)^{\mathcal{I}}(\mathrm{S} 8, \mathrm{~S} 5)=1$. For instance, for $S 6$ the supremum happens in the following case: containsWordSense ${ }^{\mathcal{I}}\left(\mathrm{S} 6\right.$, OffshoreBreakwater) $\otimes$ subsenseOf $^{\mathcal{I}}$ (Offshore Breakwater, Breakwater) $\otimes \operatorname{inSynset}^{\mathcal{I}}$ (Breakwater, S5) $=\min \{1,1,0.9\}=0.9$ is the supremum of the pairs of senses. Without our fuzzy extension, $S 5$ is as hyponym of $S 6$ as it is of $S 7$, but now we are able to quantify the degree of hyponymy. 


\begin{tabular}{|c|c|c|c|c|c|c|c|c|c|c|}
\hline & \multicolumn{7}{|c|}{ S5 } \\
\hline & & & & 0.9 & 1 & 1 & 0.8 & 0.2 & 0.5 & 0.9 \\
\hline & & & & 1 & 2 & 3 & 4 & 5 & 6 & 7 \\
\hline & & & & $\underline{\text { breakwater }}$ & groin & groyne & mole & bulwark & seawall & jetty \\
\hline \multirow{2}{*}{ S6 } & 1 & 8 & $\underset{\text { breakwater }}{\text { offshore }}$ & 1 & 0.7 & 0.7 & 0.6 & 0.2 & 0.2 & 0.6 \\
\hline & 0.9 & 9 & $\begin{array}{l}\text { detached } \\
\text { breakwater }\end{array}$ & 1 & 0.8 & 0.8 & 0.6 & 0.3 & 0.4 & 0.7 \\
\hline \multirow{2}{*}{ S7 } & 1 & 10 & $\begin{array}{l}\text { convex } \\
\text { seawall }\end{array}$ & 0.6 & 0.5 & 0.5 & 0.4 & 0.8 & 1 & 0.4 \\
\hline & 0.9 & 11 & $\begin{array}{l}\text { rigid } \\
\text { revetment }\end{array}$ & 0.5 & 0.5 & 0.5 & 0.3 & 0.8 & 0.9 & 0.4 \\
\hline \multirow{2}{*}{ S8 } & 1 & 12 & $\frac{\text { fish-tail }}{\text { groin }}$ & 0.9 & 1 & 1 & 0.8 & 0.5 & 0.5 & 0.9 \\
\hline & 1 & 13 & $\frac{\text { Y-shaped }}{\text { groin }}$ & 0.9 & 1 & 1 & 0.8 & 0.5 & 0.5 & 0.9 \\
\hline
\end{tabular}

Fig. 4. Similarity degrees between senses $\left(\alpha_{i, j}\right.$, shown in plain text) and prototype resemblance degrees between senses and synsets $\left(\beta_{k, S_{l}}\right.$, shown in italics). The white numbers in shaded cells denote the sense identification number. The labels $S 5, S 6$, etc. denote the synset identification numbers. (Identification numbers are ours.)

Equation 1 is equivalent to the following axiom, which specifies how to compute the minimal degree of a synset being an hypernym of another synset:

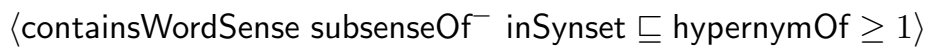

Given the fuzzy ontology $\mathcal{K}$ representing WordNet and EcoLexicon knowledge, we are interested in solving two reasoning tasks:

- Computing the minimal degree for a synset sy1 being a (possibly non-direct) hyponym of a synset sy2. This can be solved by computing the Best Degree Bound (BDB) bdb $(\mathcal{K},($ sy 1, sy 2$)$ : hyponymOf 3 .

- Computing the minimal degree for a synset sy1 being a (possibly non-direct) hypernym of a synset sy2 using $b d b(\mathcal{K},($ sy 1, sy 2$)$ : hypernymOf $)$.

Other reasoning tasks can be considered, such as the computation of the minimal degree for a word sense being similar to another word sense (either in the same or in other synset). This remains as a future work.

There are two possibilities to reason under this approach. The first one is to use an appropriate fuzzy ontology reasoner such as DeLorean, which is, to the best of our knowledge, the only existing reasoner that supports the expressivity of fuzzy $\mathcal{S} \mathcal{R}^{-} \mathcal{I}$. We will discuss here another option: creating an ad hoc crisp representation with the same semantics of the fuzzy ontology (following the same approach as for the reduction procedure internally performed by DeLorean) that can be processed with any classical ontology reasoner, such as Pellet or Hermit.

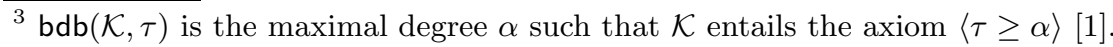




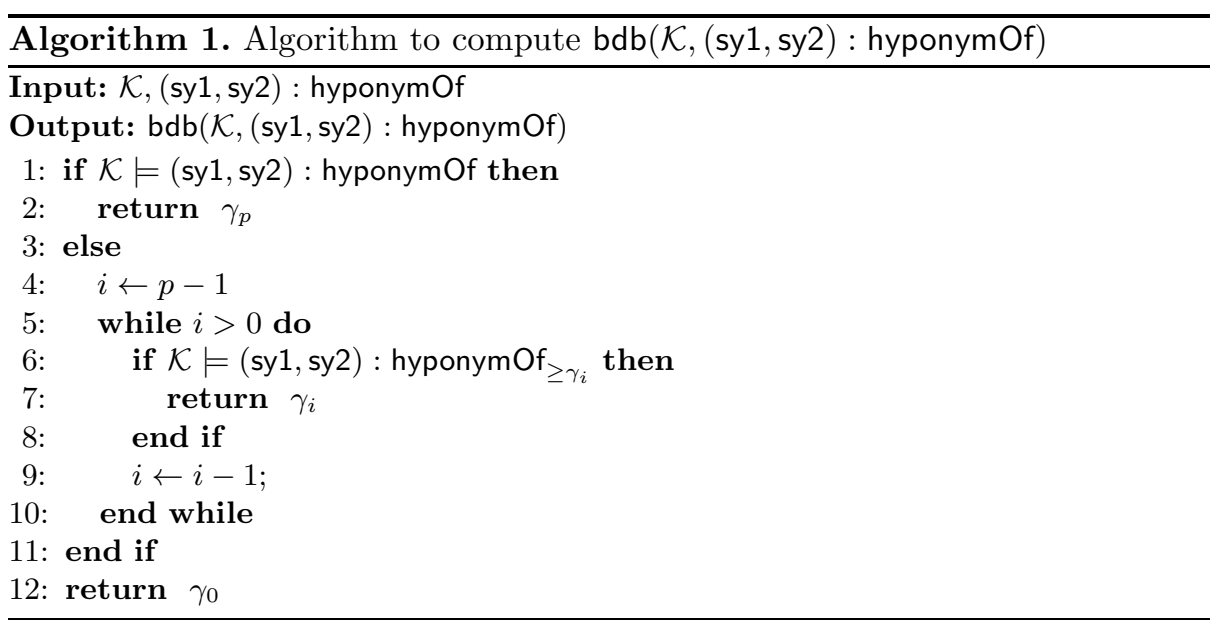

- For each $i \in\{1,2, \ldots, p-1\}$, create the properties containsWordSense ${ }_{\geq i}$, inSynset $_{\geq i}$, hyponymOf $\mathrm{f}_{\geq i}$, hypernymOf , subsenseOf $_{\geq i}$.

- For each $i \in\{1,2, \ldots, p-1\}$, assert that subsenseOf $\geq_{i}$ is transitive.

- For each $i \in\{1,2, \ldots, p-1\}$, state that containsWordSense $\geq_{i}$ is the inverse of $_{\text {inSynset }}$, $_{i}$, and that hyponymOf ${ }_{\geq i}$ is the inverse of hypernymOf ${ }_{\geq i}$.

- For each $j \in\{1, \ldots, p-2\}$ and each $S \in$ \{containsWordSense, inSynset, hyponymOf, hypernymOf, subsenseOf $\}$ add the axioms: $\mathrm{S}_{\geq j+1} \sqsubseteq \mathrm{S}_{\geq j}$.

- For each $S \in$ \{containsWordSense, inSynset, hyponymOf, hypernymOf, subsenseOf $\}$, add the axiom: $\mathrm{S}_{\geq p-1} \sqsubseteq \mathrm{S}$.

- For each $i \in\{1,2, \ldots, p-1\}$, add the following axioms (encoding part of Equation 11): containsWordSense ${ }_{\geq i} \mathrm{~S}_{\geq 1}$ inSynset $_{\geq_{i}} \sqsubseteq$ hyponymOf ${ }_{\geq i}$.

- Add the following axiom containsWordSense subsenseOf inSynset $\sqsubseteq$ hyponymOf.

- To relate two individuals with degree $\alpha$ via some relation $S$ $\in\{$ containsWordSense, inSynset, hyponymOf, hypernymOf, subsenseOf $\}$, rather use the crisp relation $S_{\geq \alpha}$ if $\alpha \neq \gamma_{p}$, or the crisp relation $S$ otherwise.

- Given our crisp ontology $\mathcal{K}$ which extends WordNet, the $\operatorname{BDB}$ bdb $(\mathcal{K},($ sy 1 , sy2) : hyponymOf) can be computed using Algorithm 1 .

\section{Conclusions and Future Work}

The example in the previous section shows that the fuzzy ontology allows us to extend WordNet with a more fine-grained description of synsets and hyponymic relations, which is necessary in specialized knowledge, and to link it with EcoLexicon. According to the presented ad hoc reduction to a crisp ontology, it is not required to use specific languages or reasoners for fuzzy ontologies. The fuzzy ontology facilitates the addition of new synsets and new general axioms, such as the one that is used to automatically infer the degree of the hyponymic relations. 
It would also be possible to add other axioms to calculate the similarity degree between arbitrary terms, but this remains as a matter for future research. Moreover, we could even consider the fuzzification of other relations in WordNet to better represent antonymy, linguistic variations, etc. Last but not least, extending WordNet to include imprecise knowledge requires a considerable effort to define the synset membership degree of word senses and the semantic similarity between word senses. We assume that experts can assign these values, but it may not be desirable or even possible in several cases, even though they would only be necessary in the synsets representing specialized knowledge. It would be interesting to study how these degrees can be obtained from the compared analysis of terminological resources (matching between WordNet and EcoLexicon semantic networks, lexical comparison of WordNet glosses and EcoLexicon definitions), and the development of corpus-based text processing procedures.

\section{References}

1. Bobillo, F., Delgado, M., Gómez-Romero, J.: Crisp representations and reasoning for fuzzy ontologies. International Journal of Uncertainty, Fuzziness and Knowledge-Based Systems 17(4), 501-530 (2009)

2. Bobillo, F., Delgado, M., Gómez-Romero, J.: DeLorean: A reasoner for fuzzy OWL 2. Expert Systems with Applications 39(1), 258-272 (2012)

3. Gómez-Pérez, A., Corcho, O., Fernández-López, M.: Ontological Engineering: with examples from the areas of Knowledge Management, e-Commerce and the Semantic Web. Springer (2004)

4. Lai, L.F., Wu, C.C., Lin, P.Y., Huang, L.T.: Developing a fuzzy search engine based on fuzzy ontology and semantic search. In: Proceedings of the 20th IEEE International Conference on Fuzzy Systems (FUZZ-IEEE 2011), pp. 2684-2689 (2011)

5. León Araúz, P., Reimerink, A., Faber, P.: Environmental knowledge in EcoLexicon. In: Jassem, K., Fuglewicz, P., Piasecki, M. (eds.) Proceedings of the Computational Linguistics Applications Conference (CLA 2011), Jachranka, Poland, pp. 9-16 (2011)

6. Lukasiewicz, T., Straccia, U.: Managing uncertainty and vagueness in description logics for the semantic web. Journal of Web Semantics 6(4), 291-308 (2008)

7. Miller, G.A.: WordNet: A lexical database for English. Communications of the ACM 38(11), 39-41 (1995)

8. Oltramari, A., Gangemi, A., Guarino, N., Masolo, C.: Restructuring WordNet's top-level: The OntoClean approach. In: Simov, K. (ed.) Proceedings of the Ontologies and Lexical Knowledge Bases Workshop (OntoLex'2), pp. 17-26 (2002)

9. Osherson, D., Smith, E.: On the adequacy of prototype theory as a theory of concepts. Cognition 9, 35-58 (1981)

10. Rosch, E.: Principles of categorization. In: Rosch, E., Lloyd, B. (eds.) Cognition and Categorization. Lawrence Erlbaum Associates (1978)

11. Staab, S., Studer, R.: Handbook on ontologies. Springer (2009)

12. van Assem, M., Gangemi, A., Schreiber, G.: RDF/OWL representation of WordNet (W3C Working Draft) (June 2006), http://www.w3.org/TR/wordnet-rdf

13. Wittgenstein, L.: Philosophical Investigations. Basil Blackwell (1953)

14. Zadeh, L.: A note on prototype theory and fuzzy sets. Cognition 12, 291-297 (1982) 